\title{
Aberration Corrector Tuning with Machine-Learning-Based Emittance Measurements and Bayesian Optimization
}

\author{
Chenyu Zhang ${ }^{1}$, Zhaslan Baraissov ${ }^{2}$, Cameron Duncan ${ }^{3}$, Adi Hanuka ${ }^{4}$, Auralee Edelen ${ }^{4}$, Jared Maxson ${ }^{3}$ and \\ David Muller ${ }^{5}$
}

${ }^{1}$ School of Applied and Engineering Physics, Cornell University, Ithaca, New York, United States, ${ }^{2}$ School of Applied and Engineering Physics, Cornell University, United States, ${ }^{3}$ Department of Physics, Cornell University, United States, ${ }^{4}$ SLAC National Accelerator Laboratory, United States, ${ }^{5}$ School of Applied and Engineering Physics, Cornell University, Ithaca, NY, USA, Ithaca, New York, United States

Aberration-corrected scanning transmission electron microscopes (STEM) use a series of multipole magnets to generate a sub-Ångstrom-sized electron beam for atomic resolution imaging and chemical composition mapping ${ }^{1}$. A new scheme for aberration corrector alignment is proposed, including both new ways of beam quality measurement and aberration corrector control. The new scheme targets fully automated corrector alignment to achieve microscope tuning with greater speed and less human bias.

For beam quality measurements, we trained a convolutional neural network (CNN) to determine the beam emittance $^{2}$ from a single Ronchigram, as shown in Figure 1(a). Beam emittance is a single variable that characterizes the volume occupied by the beam in the phase space. Emittance is convex against the aberration coefficients, and proportional to the root mean square of the phase error, as shown in Figure 1(b). Both indicate that beam emittance is a single robust objective for aberration correction, and avoids the cusp-like instabilities of the individual aberration coefficients ${ }^{3}$. The emittance would be a conserved quantity without the introduction of aberrations, and aberration correction can be guided by minimizing the emittance growth. We trained a CNN with a VGG16 architecture using simulated Ronchigram-emittance pairs to predict emittance from a single Ronchigram. The Ronchigrams are simulated using a random phase plate following ref ${ }^{4}$, with emittance calculated from the probe profile. Validation results of the CNN shown in Figure 1(c) suggest that it can predict the correct emittance value down to about $0.1 \mathrm{pm} \cdot \mathrm{rad}$ except when the noise level is high as a result of too-short an exposure time $(\sim 0.1 \mathrm{~ms})$.

We applied Gaussian Processes (GP $)^{5}$, a Bayesian approach, to search for optimal corrector parameters. A general particle tracer $(\mathrm{GPT})^{6}$ simulation model was used to simulate the electron probe at the sample for STEM column with a simple hexapole corrector ${ }^{7}$. The ray diagram of the GPT model with components marked is shown in Figure 2(a). The beam emittance calculated from the phase space distribution of all the electrons in the GPT output has been used as GP's objective function to be minimized. Figure 2(b) shows the emittance evolution over 100 iterations during the GP optimization of a partial alignment controlling the current of the two hexapole elements, for 10 random starting points. The beam quality improvement from uncorrected state to the corrected state guided by GP can be seen in the Ronchigram shown in Figure 2(c) and (d), where the half-angle of the flat area increased from about $10 \mathrm{mrad}$ to about $27 \mathrm{mrad}$.

Overall, we have shown a new method to tune the aberration corrector using emittance as an alternate metric to measure beam quality and a combination of machine learning tools to monitor and optimize the beam. The emittance prediction by CNN was tested on simulated Ronchigrams and found to be robust over a wide range of experimental conditions. Corrector tuning with GP has been tested on a GPT simulation model and effectively tuned the corrector to eliminate spherical aberration in the system ${ }^{8}$. 

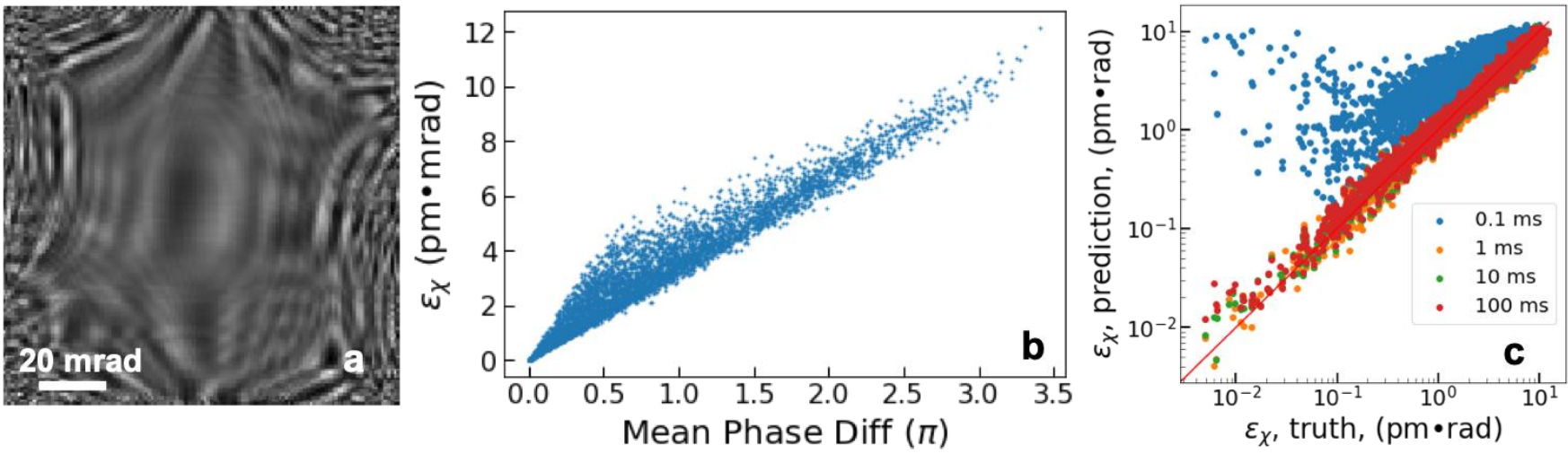

Figure 1. (a) simulated Ronchigram with noise, (b) relationship between emittance and mean phase difference, (c) emittance predicted by CNN vs. the truth emittance under different noise levels from different acquisition time.
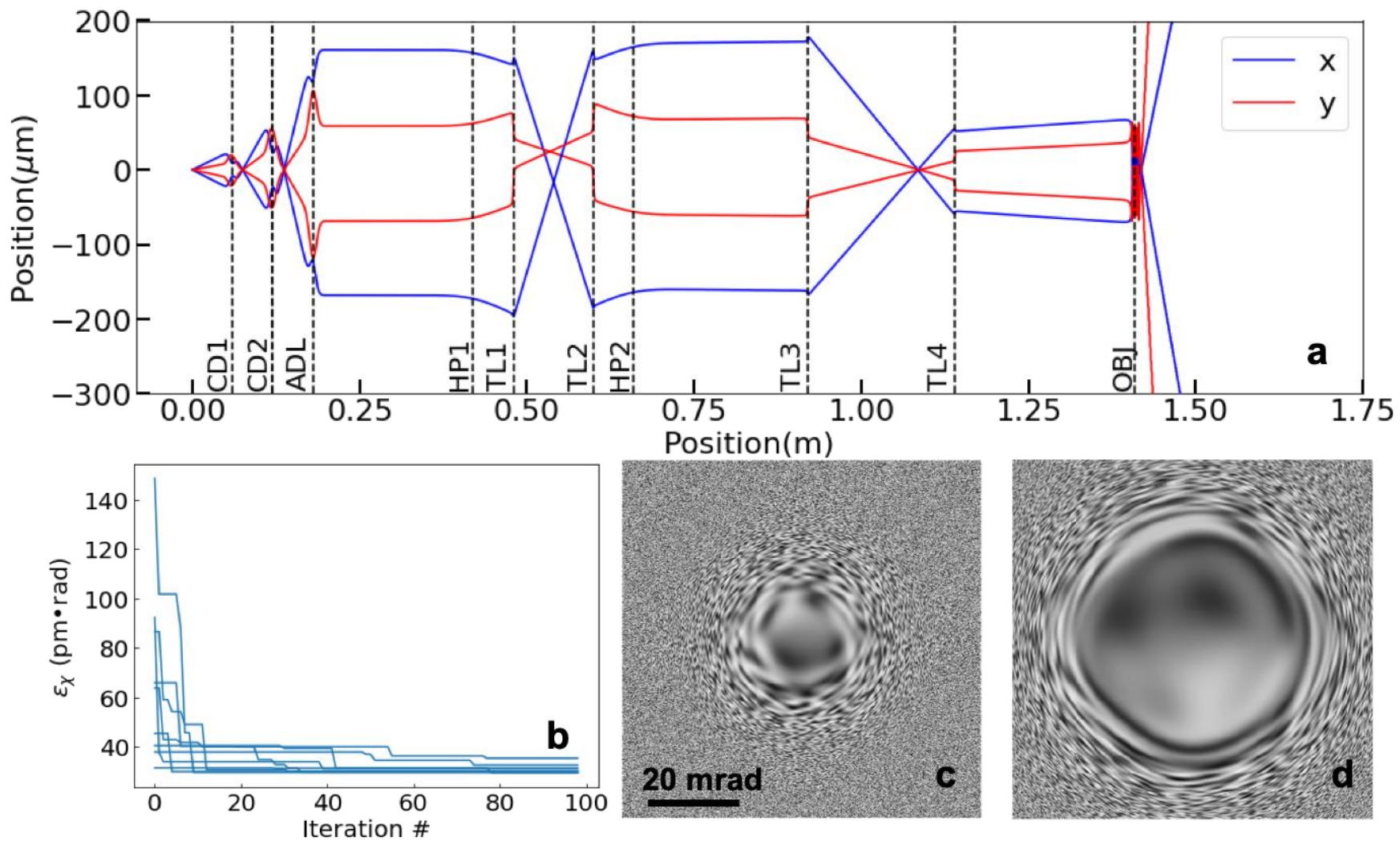

Figure 2. (a) Ray diagram of GPT simulation model contains condenser lenses (CD), hexapole elements (HP), transfer lenses (TL), adapter lens (ADL), and objective lens (OBJ). (b) emittance minimization performed by GP over 100 iterations starting from 30 random starting points. (c) and (d) comparison of GPT simulated Ronchigrams without HPs and with HP currents optimized by GP.

\section{References}

1. Muller, D. A. Structure and bonding at the atomic scale by scanning transmission electron microscopy. Nat. Mater. 8, 263-270 (2009).

2. Floettmann, K. Some basic features of the beam emittance. Phys. Rev. Spec. Top. - Accel. Beams 6, 80-86 (2003). 
3. Schramm, S. M., Van Der Molen, S. J. \& Tromp, R. M. Intrinsic instability of aberration-corrected electron microscopes. Phys. Rev. Lett. 109, 1-5 (2012).

4. Schnitzer, N., Sung, S. H. \& Hovden, R. Optimal STEM Convergence Angle Selection Using a Convolutional Neural Network and the Strehl Ratio. Microsc. Microanal. 26, 921-928 (2020).

5. Duris, J. et al. Supplemental Material for Bayesian Optimization of a Free-Electron Laser Gaussian fit Raster scan. 1-4 (2020).

6. van der Geer, S. B. \& de Loos, M. J. General Particle Tracer (v3.1). 1-202 (2009).

7. Müller, H., Uhlemann, S., Hartel, P. \& Haider, M. Advancing the hexapole Cs-corrector for the scanning transmission electron microscope. Microsc. Microanal. 12, 442-455 (2006).

8. Funded by the Center for Bright Beams, an NSF STC (NSF PHY-1549132). 